Hautarzt 2009 - 60:263-263

DOI 10.1007/s00105-009-1746-9

Online publiziert: 26. März 2009

๑) Springer Medizin Verlag 2009

\author{
D. Petzoldt \\ Ehem. Direktor der Universitäts-Hautklinik Heidelberg
}

\title{
Gedanken zum Jubiläum
}

Die Dermatologie ist in einem ständigen Wandel begriffen. Längst steht die konservative Dermatologie mit ihrer vorwiegend externen Therapie nicht mehr allein im Zentrum unseres Faches. Teilgebiete, die früher eher peripher angesiedelt waren, stehen heute gleichberechtigt daneben.

Als Beispiele seien die operative Dermatologie, die Allergologie und Immunologie sowie die kosmetische Dermatologie genannt. Mehr und mehr an Boden gewinnt die molekulare Dermatologie, sei es auf dem Gebiet der Diagnostik und der Therapie, sei es im Bereich der Dermatoonkologie oder der entzündlichen Dermatosen.

Das bedeutet, dass sich der Dermatologe in einem lebenslangen Lernprozess befindet und seine Kenntnisse und Fähigkeiten ständig den sich ändernden Gegebenheiten anpassen muss.

Ist die Beschäftigung mit der Dermatologie in den letzten Jahrzehnten eigentlich schwieriger geworden? Eine schwer zu beantwortende Frage! Natürlich ist das Spektrum unseres Faches heute vielfältiger und der Umfang größer. Auch die Anforderungen an pathophysiologische und molekularbiologische Grundkenntnisse sind heute ungleich anspruchsvoller.

Auf der anderen Seite haben sich aber auch Vereinfachungen ergeben. Die Erkenntnisse der Grundlagenforschung haben Ursachen und pathogenetische $\mathrm{Zu}$ sammenhänge aufgeklärt und damit die Systematik und Erlernbarkeit unseres Faches wesentlich gefördert. Pharmakologische Erkenntnisse und daraus resultierende therapeutische Optionen haben die Behandlung von Hautkrankheiten unendlich effektiver und zahlreiche schwer verständliche Therapieverfahren überflüssig gemacht.
Der Hautarzt hat immer versucht, dem Wandel in der Dermatologie Rechnung zu tragen und seine Leser über neue, praxisrelevante Entwicklungen zeitnah zu informieren. Bei allem Verständnis für die Unabdingbarkeit des Wandels wurde aber eine Maxime niemals angetastet: das Primat der klinischen Dermatologie, ruhend auf ihrem naturwissenschaftlichen und nicht zuletzt morphologischen Fundament. Die über jeden Zweifel erhabene, erstklassige Qualifikation einer jeden Dermatologin und eines jeden Dermatologen zur Erkennung und Behandlung von Hautkrankheiten muss das höchste Ziel unseres Faches und damit auch der Zeitschrift Der Hautarzt bleiben.

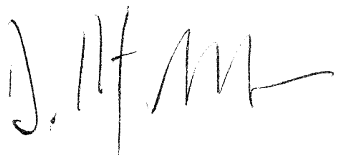

Detlef Petzoldt

Ehrenherausgeber

\section{Korrespondenzadresse \\ Prof. Dr. D. Petzoldt}

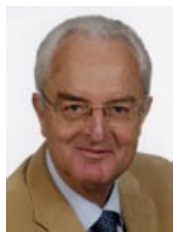

Ehem. Direktor der Universitäts-Hautklinik Heidelberg Voßstr. 2, 69115 Heidelberg 